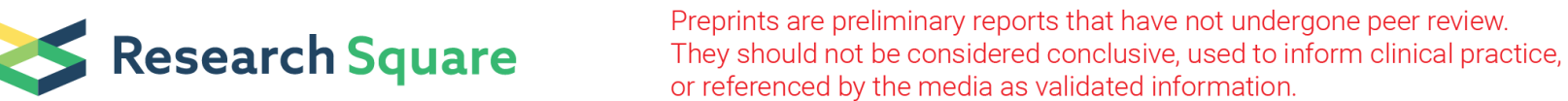

\section{QALYs and DALYs are Better with Concurrent ChemoRT than Induction Chemo Followed by ChemoRT in Nasopharyngeal Carcinoma.}

\section{Mary R Nittala}

University of Mississippi Medical Center https://orcid.org/0000-0002-9755-5672

Madhava R. Kanakamedala

University of Mississippi Medical Center

Eswar K. Mundra

University of Mississippi Medical Center

Williams C. Woods

University of Mississippi Medical Center

Maria L. Smith

University of Mississippi Medical Center

Robert D. Hamilton

University of Mississippi Medical Center

Gina D Jefferson

University of Mississippi Medical Center

\section{Lana Jackson}

University of Mississippi Medical Center

\section{Satyaseelan Packianathan}

University of Mississippi Medical Center

Srinivasan Vijayakumar ( $\sim$ svijayakumar@umc.edu )

University of Mississippi Medical Center https://orcid.org/0000-0002-1678-9451

\section{Research article}

Keywords: Quality-adjusted life year, disability-adjusted life year, Nasopharyngeal Carcinoma

Posted Date: August 26th, 2020

DOI: https://doi.org/10.21203/rs.3.rs-61627/v1

License: (c) (1) This work is licensed under a Creative Commons Attribution 4.0 International License.

Read Full License 


\section{Abstract}

Background: As traditional measures such as overall survival (OS) or disease-free survival (DFS) alone does not give a holistic view of the outcomes of a treatment paradigm, we determine to add the evidence of quality-adjusted life year (QALY) and disability-adjusted life year (DALY) to the outcomes of the nasopharyngeal carcinoma patients (NCP) treated with definitive chemoradiation therapy (chemoRT) with or without induction chemotherapy.

Methods: This is a retrospective analysis of 85 NCPs treated at an academic state institution. The OS estimated by Kaplan-Meier method and the multivariate Cox regression model determined the covariables associated with the OS. The relationship between QALYs gained and DALYs saved were calculated from age of the disease onset, duration of the disease, quality of life (QoL) and disability weights.

Results: Of the 85 eligible NCPs of this cohort, the disease frequency distribution per the World Health Organization (WHO) classification was $41.2 \%$ for Type-l, $42.4 \%$ for Type-ll, and $16.5 \%$ for Type-III. The median follow-up (24 months). The 5-year OS of patients treated with concurrent chemoRT $v s$. induction chemo followed by concurrent chemoRT was $54.7 \mathrm{vs}$. $14.8 \%$ for WHO Type I, $60.1 \mathrm{vs}$. $58.3 \%$ for WHO Type II, and 83.3 vs. $50.0 \%$ for WHO Type III ( $p=0.029)$. The average DALYs saved with concurrent chemoRT was 12.2 years vs. 5 years for induction chemo followed by concurrent chemoRT. The average QALYs gained with concurrent chemoRT was 6.9 years vs. 3.1 years for induction chemo followed by concurrent chemoRT.

Conclusion: Patients treated with concurrent chemoRT had an increased QoL when compared to induction chemo followed by concurrent chemoRT. The average DALYs saved were higher in the patients treated with concurrent chemoRT than treated with induction chemo followed by concurrent chemoRT.

\section{Background}

In the United States, nasopharyngeal carcinoma (NPC) is a rare neoplasm of the head and neck (HN) region and accounts for only $2 \%$, of all $\mathrm{HN}$ cancers; less than 1:10,000 persons diagnosed with NPC each year and 129,000 new cases were diagnosed in 2018 [1-3]. Worldwide, however, it is the 18th and 22nd most commonly occurring cancer in men and women, respectively [4, 5]. It is more common in Asian populations such as those originating from Southern China and Southeast Asia as well as in Eskimo and North African population with annual incidence rates approaching 50 cases per 100,000 persons [6-9].

Nasopharyngeal carcinoma differs from other HN cancers in epidemiology, distant metastasis rates, and its association with the Epstein-Barr virus (EBV) [10-11]. The major source of morbidity and mortality in NPC patients comes from its high risk of distant metastasis. The World Health Organization (WHO) has classified NPC into 3 types, based on histology: keratinizing squamous cell carcinoma (Type I), differentiated non-keratinizing carcinoma (Type II) and undifferentiated non-keratinizing carcinoma (Type 
III) $[13,14]$. In the US, about $25 \%$ of NPC patients have keratinizing carcinoma while $12 \%$ are differentiated non-keratinizing and $63 \%$ have the undifferentiated non-keratinizing histology [14].

Treatment for NPC depends upon the tumor stage and the patient's performance status [15]. Because of the operative morbidity associated with the anatomic location of these tumors, they are traditionally treated with radiation therapy (RT) $[15,16]$. Both non-keratinizing carcinomas (Type II \& III) respond better to radiation compared to the keratinizing carcinoma (Type I). The 5-year overall survival rates for nonkeratinizing NPC is $51 \%$ vs. $6 \%$ for the keratinizing NPC [17]. Previous studies have suggested that OS rates of NPC patients were not significantly improved by induction chemo followed by concurrent chemoRT compared to concurrent chemoRT [18], and could even worsen the survival of stage II NPC patients [19].

A patient's pre-treatment health-related quality of life (HRQoL) has been shown to be a significant independent predictor of locoregional disease control in $\mathrm{HN}$ cancer patients [20]. Thus, an understanding of the contribution of various treatment modalities to a patient's QoL is very important as it because it helps to evaluate the type of treatment the $\mathrm{HN}$ cancer patients may need, and also identify impairments in the ability to tolerate a specific treatment approach [21, 22].

Traditionally, QoL is often evaluated using health survey questionnaires such as the Quality of Life Questionnaire C30 (QLQ-C30) or the EORTC QLQ Head and Neck Cancer-Specific Module (H\&N35) [2325]. When questionnaires are unavailable, QoL is often estimated by alternative methods of qualityadjusted life year (QALY) and disability-adjusted life year (DALY) [26, 27].

QALY captures both the impact of a treatment on a patient's length of life and also its impact on their HRQoL [28]. DALY is a summary measure of public health widely used to quantify the burden of disease [29]. In this study, we used QALYs and DALYs to assess the impact of QoL [30] on survival outcomes of NPC patients treated with concurrent chemoRT with or without induction chemotherapy.

\section{Methods}

\section{Patients}

The study subjects were 96 NPC patients diagnosed and treated between 1994 and 2018 at the University of Mississippi Medical Center (UMMC), Jackson, MS. Institutional review board approval was obtained for the retrospective analysis and a browser-based database- research electronic data capture (RedCap)was used to gather and store the patient information. Written consent was waived secondary to the retrospective nature of the study and patient identifiers were removed before the data were extracted for analysis. Nine patients with an unknown WHO class of tumor were excluded from the study and hence, 85 patients were included in the final analyses.

\section{Treatment Methods}


Fifty-one patients $(60 \%)$ received concurrent chemoRT consisted of weekly Cisplatin $\left(40 \mathrm{mg} / \mathrm{m}^{2}\right)$ during radiotherapy for a maximum of 7 cycles, beginning on the first day of radiotherapy. Twenty patients (23.5\%) received induction chemo with Docetaxel, Cisplatin, and Fluorouracil (TPF) followed by concurrent chemoRT as described above, while 14 patients $(16.5 \%)$ received no treatment.

\section{QoL assessments}

QALY: Is a measure of the value of health outcome and combines length and QoL into a single unit. It is calculated simply by multiplying the duration of time spent in a health stage by the

HRQoL weighting associated with that health state.

DALY: Is a common measurement unit for morbidity and mortality. It is composed of years lived with the disability (YLD) and years of life lost (YLL) due to premature mortality associated with that disability.

$D A L Y=Y L D+Y L L$

$Y L D=$ number of cases $x$ duration till remission (or) death $x$ disability weight (DW)

$Y L L=$ number of deaths $x$ life expectancy $(L E)$ at the age of death

DW is a scale from zero (perfect health) to one (worst possible health state) and LE was obtained from the actuarial life tables.

\section{Statistical analysis}

The overall survival (OS) was defined by the number of days from the date of initial diagnosis until the date of death or the last contact. The censored cases included patients without death at the time of the last follow up. The Kaplan-Meier method was used to estimate the OS rates and the univariate significance of differences among survival curves was calculated by the log-rank test. The co-variables associated with the OS were determined by the multivariate Cox regression model. Hazards ratio (HR) was used to estimate the time-to-event outcome with associated $95 \%$ confidence intervals (Cls) and $P$ values $\leq 0.05$ were considered statistically significant. Average QALYs gained and average DALYs saved were calculated for all the variables stratified by the tumor histology. The SPSS 24.0 software was used for data analyses.

\section{Results}

\section{Patient Characteristics}

In this 85 NPC cohort, there were 55 (64.7\%) male patients and 30 (35.3\%) female patients. The median age was 56 years (range, 19- 86 years). Out of the 85 NPC patients, $35(41.2 \%)$ had WHO type I disease, 36 (42.4\%) had WHO type II disease and 14 (16.5\%) had WHO type III disease. Fifty- seven (67.1\%) were insured while $28(32.9 \%)$ had no insurance. More than half of the patients $60(70.6 \%)$ had a history of 
smoking and the use of alcohol was fifty-fifty in this group. The stage distribution in this cohort was Stage I (5.9\%), Stage II (9.4\%), Stage III (16.5\%), Stage IV (52.9\%) and Unknown Stage (15.3\%). Fifty-one (60\%) were treated with concurrent chemoRT, 20 (23.5\%) with induction chemo followed by the chemoRT and $14(16.5 \%)$ NPC patients were hospice (Table- 1 ). 
Table-1. Demographic \& Treatment description of the Nasopharyngeal Study Population by Histology -WHO classification

Variable

Male

Female

AGE

$<35$ years

35 - 60 years

$>60$ years

\section{ETHNICITY}

African American

Caucasian

Other Ethnicity

INSURANCE

Medicaid

Medicare

Private

Self-pay

DISTANCE

$<30$ miles

30 - 75 miles

$>75$ miles

\section{SMOKING}

Smoker

Non-Smoker

\section{GENDER/SEX}

$$
\begin{aligned}
& \text { WHO T } \\
& \mathrm{n}=35
\end{aligned}
$$

$(41.2 \%)$
WHO Type II $n=36(42.4 \%)$
WHO Type All patients III

$\mathrm{n}=14$

$(16.5 \%)$ pvalue

$\mathrm{n}=85$

$(100 \%)$ 


\section{ALCOHOL}

\section{Drinker}

Non-Drinker

TUMOR STAGE

\begin{tabular}{|c|c|c|c|c|c|}
\hline Stage I & $2(5.7 \%)$ & $1(2.8 \%)$ & $2(14.3 \%)$ & $5(5.9 \%)$ & \\
\hline Stage II & $3(8.6 \%)$ & $5(13.9 \%)$ & $0(0.0 \%)$ & $8(9.4 \%)$ & \\
\hline Stage III & $6(17.1 \%)$ & $\begin{array}{c}8 \\
(22.2 \%)\end{array}$ & $0(0.0 \%)$ & $14(16.5 \%)$ & \\
\hline Stage IV & $21(60.0 \%)$ & $19(52.8 \%)$ & $5(35.7 \%)$ & $45(52.9 \%)$ & \\
\hline Unknown Stage & $3(8.6 \%)$ & $3(8.3 \%)$ & $7(50.0 \%)$ & $13(15.3 \%)$ & 0.005 \\
\hline \multicolumn{6}{|l|}{ TREATMENT } \\
\hline Concurrent chemoRT & $21(60.0 \%)$ & $24(66.7 \%)$ & $6(42.9 \%)$ & $51(60.0 \%)$ & \\
\hline $\begin{array}{l}\text { Induction chemo + } \\
\text { chemoRT }\end{array}$ & $9(25.7 \%)$ & $8(22.2 \%)$ & $3(21.4 \%)$ & $20(23.5 \%)$ & \\
\hline Hospice & $5(14.3 \%)$ & $4(11.1 \%)$ & $5(35.7 \%)$ & $14(16.5 \%)$ & 0.292 \\
\hline
\end{tabular}

$\mathrm{n}=$ number, $\mathrm{WHO}=$ World Health Organization, chemoRT $=$ chemo radiation therapy, Induction chemo= Induction chemotherapy.

\section{QoL data}

The average QALYs gained and average DALYs saved for different variables stratified by the histology of the NPC in the study population (Table 2). The average LE for this 85- patient NPC cohort was 34.51 years, average DALYs saved with treatment was 20.06 years and average QALYs gained with treatment was 11.77 years.

The DALYs saved with concurrent chemoRT was 12.2 years vs 5 years with induction chemo followed by concurrent chemoRT. The QALYs gained with concurrent chemoRT was 6.9 years vs. 3.1 years with induction chemo followed by concurrent chemoRT.

\section{Survival Outcomes}

The median follow up for this study population was 24 months. The 5-year OS of WHO Type I was $34.8 \%$, Type II $58 \%$ and Type III $77.1 \%$ ( $p=0.042)$. The median survival of WHO type I was 34 months, Type II 194 months and Type III 123 months (shown in Fig. 1). 
The OS curve for different tumor stages are represented (shown in Fig. 1). For all histologies the 5-year OS for Stage I was $80.0 \%$, Stage II $57.1 \%$, Stage III $52.6 \%$, Stage IV $44.5 \%$ and Stage Unknown $64.5 \%$ ( $p=$ $0.321)$.

\section{Univariate Analyses}

In the univariate analyses, the variables of gender, age, ethnicity, insurance, distance traveled to the treatment facility, alcohol history, and the treatment modality was significantly associated with the OS (Table 3).

The OS curves for the treatment modalities used are represented (shown in Fig. 2). Five-year OS of NCPS treated by concurrent chemoRT for WHO Type I $54.7 \%$, WHO Type II $60.1 \%$, WHO Type III $83.3 \%$ ( $p=$ 0.029). Five-year OS of NCPs treated by induction chemo followed by concurrent chemoRT for WHO Type I $14.8 \%$, WHO Type II $58.3 \%$ and WHO Type III $50.0 \%(p=0.029)$. 


\begin{tabular}{|c|c|c|}
\hline Variable & Ave QALYs Gained & Ave DALYs Saved \\
\hline \multicolumn{3}{|l|}{ GENDER/SEX } \\
\hline Male & 11.63 & 20.19 \\
\hline Female & 10.06 & 18.53 \\
\hline \multicolumn{3}{|l|}{ AGE } \\
\hline$<35$ years & 17.01 & 26.01 \\
\hline $35-60$ years & 12.35 & 21.37 \\
\hline$>60$ years & 7.67 & 15.38 \\
\hline \multicolumn{3}{|l|}{ ETHNICITY } \\
\hline African American & 11.53 & 20.09 \\
\hline Caucasian & 11.51 & 19.62 \\
\hline Other Ethnicity & 4.06 & 15 \\
\hline \multicolumn{3}{|l|}{ INSURANCE } \\
\hline Medicaid & 13.55 & 22.75 \\
\hline Medicare & 8.67 & 15.77 \\
\hline Private & 8.33 & 16.63 \\
\hline Self-pay & 12.17 & 21.22 \\
\hline \multicolumn{3}{|l|}{ DISTANCE } \\
\hline$<30$ miles & 11.49 & 20.73 \\
\hline 30 - 75 miles & 13.1 & 20.35 \\
\hline$>75$ miles & 10.21 & 18.78 \\
\hline \multicolumn{3}{|l|}{ SMOKING } \\
\hline Smoker & 10.86 & 19.43 \\
\hline Non-Smoker & 11.62 & 20.04 \\
\hline \multicolumn{3}{|l|}{ ALCOHOL } \\
\hline Drinker & 11.03 & 19.84 \\
\hline Non-Drinker & 11.13 & 19.36 \\
\hline TUMOR STAGE & & \\
\hline
\end{tabular}




\begin{tabular}{|c|c|c|c|c|}
\hline Stage I & & 7.71 & & 15.67 \\
\hline Stage II & & 8.07 & & 17.28 \\
\hline Stage III & & 11.2 & & 18.52 \\
\hline Stage IV & & 12.4 & & 21.27 \\
\hline Unknown Stage & & 9.49 & & 17.95 \\
\hline \multicolumn{5}{|l|}{ TREATMENT } \\
\hline Concurrent chemoRT & 10.93 & & 19.9 & \\
\hline Induction chemo + chemoRT & 12.61 & & 20.22 & \\
\hline Hospice & 9.75 & & 17.66 & \\
\hline
\end{tabular}

Ave= average, $Q A L Y=$ quality-adjusted life year, $D A L Y=$ disability-adjusted life year, chemoRT $=$ chemo radiation therapy, Induction chemo $=$ induction chemotherapy.

\begin{tabular}{|ll|}
\hline \multicolumn{2}{|l|}{ Table -3 . Univariate analysis by tumor histology } \\
\hline Variable & \multicolumn{1}{l|}{ p Value } \\
\hline GENDER/SEX & 0.013 \\
\hline AGE & 0.013 \\
\hline ETHNICITY & 0.042 \\
\hline INSURANCE & 0.026 \\
\hline DISTANCE & 0.041 \\
\hline SMOKING & 0.960 \\
\hline ALCOHOL & 0.010 \\
\hline TUMOR STAGE & 0.321 \\
\hline TREATMENT & 0.029 \\
\hline
\end{tabular}




\begin{tabular}{|lcc|}
\hline \multicolumn{2}{|c|}{ Table -4. Multivariate Cox regression analysis } & \\
\hline Variable & HR $(95 \% \mathrm{Cl})$ & p value \\
\hline GENDER/SEX & $0.52(0.24-1.15)$ & 0.111 \\
\hline AGE & $0.84(0.48-1.47)$ & 0.557 \\
\hline ETHNICITY & $0.87(0.50-1.53)$ & 0.879 \\
\hline INSURANCE & $1.13(0.85-1.49)$ & 0.389 \\
\hline DISTANCE & $0.87(0.58-1.29)$ & 0.496 \\
\hline ALCOHOL & $1.40(0.71-2.78)$ & 0.324 \\
\hline TREATMENT & $0.88(0.48-1.59)$ & 0.682 \\
\hline HISTOLOGY & $0.61(0.39-0.94)$ & 0.027 \\
\hline
\end{tabular}

$95 \%=95$ percentage, $\mathrm{Cl}=$ confidence interval

\section{Multivariate Analyses}

The variables which were significantly associated with survival outcomes were included in the Cox proportional hazards regression model (Table 4). In the multivariate analyses, only tumor histology was significantly associated with OS, with HR of $0.61(95 \% \mathrm{Cl} 0.39-0.94), p=0.027$.

\section{Discussion}

Nasopharyngeal carcinoma is a rare neoplasm of the $\mathrm{HN}$ in the United States and accounts for only $2 \%$ of all the $\mathrm{HN}$ cancers [1-3]. Our data for this cohort of patients, accounting for $2.4 \%$ of all the patients in our HN cancer database, is consistent with the literature. Patients with NPC are mostly males with a known history of tobacco and heavy alcohol use $[30,31]$ and our data reflects what has been previously reported as $65 \%$ were males with $71 \%$ had a known history of tobacco and $51 \%$ had a history of heavy alcohol use. Nasopharyngeal carcinoma is more common in Asians/Pacific Islanders, who are diagnosed 6 times more frequently than Caucasian and Hispanic people [32], and in whom the disease can occur at any age, including children, while about $50 \%$ of people with the disease are 55 or younger. Our patient cohort consisted of $39 \%$ Caucasian and 55\% African Americans, which is most likely due to our regional demographics, while $53 \%$ of the cohort were younger than 60 years.

Generally, the NPC histology distribution in the United States is keratinizing carcinoma (Type I) $25 \%$, differentiated non-keratinizing (Type II) $12 \%$ and undifferentiated non-keratinizing (Type III) $63 \%$. In our cohort, these histologies, however, were represented by $41 \%, 42 \%$, and $17 \%$, respectively. It is possible that these differences are due to the ethnic demographics of our patient population with very few Asian/Pacific islanders represented. In the literature, the reported 5-year OS for non-keratinizing NPC is 
$51 \%$ vs. $6 \%$ for keratinizing NPC [17]. In our cohort though, the 5 -year OS were $66 \%$ vs. $38 \%$ with a median survival of 24 months.

It is established that NPC patients have a higher likelihood of living cancer-free for an extended period of time with treatment methods that utilize radiation therapy (RT) alone or in combination with chemotherapy [33-35]. Many reports have suggested that treatments delivered with more advanced RT techniques show a significant trend toward improving QoL outcomes [36, 37]. In a prospective randomized trial comparing the QoL of NPC patients treated with 2DRT $v s$. IMRT, it was shown that IMRT significantly improved salivary flow after RT, although no other scale being measured significantly improved, except physical and role functioning, as assessed by the EORTC QLQ-C30 38, 39]. Some studies have noted that the use of 3DchemoRT/IMRT reduced the HN-related symptom scales to some extent and thereby improved QoL [40]. Besides the RT technique used, socioeconomic status, comorbidity, and tumor site were also found to be significant prognostic predictors of a patient's HR-QoL outcome. Patients who had higher economic status, higher education levels, employed status, and fewer comorbidities tented to have better HR-QoL [41]. Some studies have reported that female gender, higher cancer stage, and combination treatment were associated with more symptoms and worse HR-QoL [42, 43]. Few studies had explored the prognostic significance of QoL in QLQ-C30 questionnaires for NPC patients. Studies conducted to evaluate the impact of replanning on the QoL during IMRT showed that replanning had both statistically and clinically significant improvements in QoL scales [44]. In our study, we found that the OS of concurrent chemoRT $v s$. induction chemo followed by concurrent chemoRT was $32.8 \%$ vs. $14.8 \%(p=0.029)$.

Many studies have previously used the EORTC health survey questionnaires to investigate the QoL of NPC patients. For our analysis, however, we were unable to estimate patient QoL using the questionnaire method as our institution started using health survey questionnaires only recently. Thus, in order to calculate QoL without available survey data, we used QALYs and DALYs as health outcome measures that account for both longevity and QoL $[26,27]$. In our investigation, we found that the average LE for our 85 NPC cohort was 34.56 years, the average DALYs saved with treatment was 20.06 years and average QALYs gained with treatment was 11.77 years. The DALYs saved with concurrent chemoRT was 12.2 years vs. 5 years with induction chemo followed by concurrent chemoRT. The QALYs gained using concurrent chemoRT was 6.9 years vs. 3.1 years with induction chemo followed by concurrent chemoRT.

As with all retrospective analyses, our study is not without its limitations. As we had no HR-QoL data available, potential selection bias is one of the limitations. The QALY approach used in this study does not explicitly incorporate equity weights, which can be a challenge when comparing treatment interventions. In order to overcome the challenges posed by using the QALY approach, we utilized an alternative method, DALY, to compare the impact of disease burden among treatment modalities.

\section{Conclusions}


Our retrospective analysis reports OS findings in NPC similar to that in previous studies by Liang et.al and Tan et.al, that is, induction chemo followed by chemoRT was not shown to improve OS compared to definitive chemoRT. In addition, our data suggest that patients treated with concurrent chemoRT had an improved QoL compared to that of patients treated with induction chemo followed by concurrent chemoRT. The average DALYs saved and average QALYs gained were higher in the patients treated with concurrent chemoRT. However, there may be some yet unidentified factors or some specific functional domains, related to the QoL which were unappreciated in this analysis.

\section{List Of Abbreviations}

ChemoRT Chemoradiation

Cl Confident Interval

DALY Disability-Adjusted Life Year

DFS Disease- Free Survival

EBV Epstein - Barr virus

HRQoL Health Related Quality of Life

HN Head and Neck

NPC Nasopharyngeal Carcinoma

OS Overall Survival

QALY Quality- Adjusted Life Year

QoL Quality of Life

RedCap Research Electronic Data Capture

Type-I Keratinizing Squamous Cell Carcinoma

Type-II Differentiated Non-Keratinizing Carcinoma

Type-III Undifferentiated Non- Keratinizing Carcinoma

UMMC University of Mississippi Medical Center

WHO World Health Organization

YLD Years Lived with the Disability 


\section{Declarations}

Ethics approval and consent to participate: The subjects (or their parents or guardians) written consent was waived by Health Insurance Portability and Accountability Act (HIPPA) due to the retrospective nature of the study and patient identifiers were removed before extracting the data.

University of Mississippi Medical Center - Institutional Review Board (IRB) Approval \#2010-0252 "Outcomes following treatment for Head and Neck Cancer"

Consent for Publication: Not applicable

Availability of data and Materials: This study does not contain any sequence/ expression data, protein/molecule characterizations, annotations, and taxonomy data to be deposited in a public repository. Patient identifiers were removed before extracting data and will be shared upon reasonable request.

Competing Interests: There is no conflict of interest present for authors relative to this research

Funding: This research did not receive any specific grant from funding agencies in the public, commercial or not-for-profit sectors.

Author's Contributions: MN: developed the concept, data collection, data analysis, writing the manuscript, drafting, final revision, editing and submission; MR, RD, GJ, LJ, SP \& SV: clinical concept; ML \& WW: editing; EM: clinical management SP \& SV: final revision, grammatical check \& editing.

Acknowledgements: Not applicable

\section{References}

1. American cancer Society. Cancer facts and figures 2019. [Available from https://www.cancer.org/cancer/nasopharyngeal-cancer/about/key-statistics.html].

2. S-HI Ou, JA Zell, A Ziogas, H Anton-Culver. Epidemiology of nasopharyngeal carcinoma in the United States: improved survival of Chinese patients within the keratinizing squamous cell carcinoma histology. Annals of Oncology. 2007; 18:29-35. https://doi.org/10.1093/annonc/mdl320.

3. L Davies, HG Welch. Epidemiology of head and neck cancer in the United States. Otolaryngol Head Neck Surg. 2006; 1353: 451-457.

4. Parkin DM, Bray F, Ferlay J, Pisani P. Global cancer statistics. Cancer J Clin 2005; 55:74-108.

5. C Fitzmaurice, C Allen, RM Barber, et al. Global Burden of Disease Cancer Collaboration. Global, regional, and national cancer incidence, mortality, years of life lost, years lived with disability, and disability-adjusted life-years for 32 cancer groups, 1990 to 2015: a systematic analysis for the global burden of disease study JAMA Oncol. 2017; 3: 524-548. 
6. M Al-Sarraf, M LeBlanc, P G Giri, K K Fu, J Cooper, T Vuong, A A Forastiere, G Adams, W A Sakr, D E Schuller, and J F Ensley. Journal of Clinical Oncology. 1998; 16: 1310-1317.

7. J. H. Ho. An epidemiology and clinical study of nasopharyngeal carcinoma. Int J Radiat Oncol Biol Phys. 1978; $4: 183$

8. Qin DX, Hu YH, Yan JH, et al. Analysis of 1379 patients with nasopharyngeal carcinoma treated by radiation. Cancer. 1988; 61:1117-1124.

9. JT Wee, TC Ha, SL Loong, et al. Is nasopharyngeal cancer really a "Cantonese cancer"? Chin J Cancer. 2010; 29: 517-526

10. N. Raab-Traub. Epstein-Barr virus in the pathogenesis of NPC. Semin Cancer Biol. 2002; 12: 431-441

11. Niedobitek G, Meru N, Delecluse HJ. Epstein-Barr virus infection and human malignancies. Int J Exp Path. $2001 ;$ 82: 149-170.

12. Petersson F. Nasopharyngeal carcinoma: A review. Semin Diagn Pathol. 2015; 32:54-73.

13. Stelow, E.B. \& Wenig, B.M. Update from the 4th Edition of the World Health Organization Classification of Head and Neck Tumours: Nasopharynx. Head and Neck Pathol .2017; 11: 16. [Available from: https://doi.org/10.1007/s12105-017-0787-0].

14. Thompson, LD. Update on nasopharyngeal carcinoma. Head Neck Pathol. 2007; 1:81-86.

15. Wang, Y, Zhang, Y, Ma, S. Racial differences in nasopharyngeal carcinoma in the United States. Cancer Epidemiol. 2013; 37:793-802.

16. F.M. Fang, W.L. Tsai, S.F. Go, et al. Implications of quantitative tumor and nodal regression rates for nasopharyngeal carcinomas after 45 Gy of radiotherapy. Int J Radiat Oncol. 2001; 50:961-969

17. Reddy, SP, Raslan, WF, Gooneratne, S, Kathuria, S, Marks, JE. Prognostic significance of keratinization in nasopharyngeal carcinoma. Am J Otolaryngol. 1995; 16:103-108.

18. L. Chen, C.S. Hu, X.Z. Chen, et al. Concurrent chemoradiotherapy plus adjuvant chemotherapy versus concurrent chemoradiotherapy alone in patients with locoregionally advanced nasopharyngeal carcinoma: a phase 3 multicenter randomized controlled trial. Lancet Oncol. 2012; 13:163-171.

19. Jin T, Zhang Q, Luo DH, et al. Concurrent chemoradiotherapy with or without induction chemotherapy for patients with sthae II nasopharyngeal carcinoma: an update. Trans Oncol, 2020; 13:25-31.

20. F. Siddiqui, T.F. Pajak, D. Watkins-Bruner, et al. Pretreatment quality of life predicts for locoregional control in head and neck cancer patients: A radiation therapy oncology group analysis. Int $\mathrm{J}$ Radiat Oncol Biol Phys. 2008; 70:353-360

21. B. Movsas, C. Scott, D. Watkins-Bruner. Pretreatment factors significantly influence quality of life in cancer patients: A Radiation Therapy Oncology Group (RTOG) analysis. Int J Radiat Oncol Biol Phys. 2006; 65:830-835

22. Karvonen-Gutierrez, CA, Ronis, DL, Fowler, KE, Terrell, JE, Gruber, SB, Duffy, SA. Quality of life scores predict survival among patients with head and neck cancer. Journal of Clinical Oncology. 2008; 26:2754-2760. 
23. Aaronson NK, Ahmedzai S, Bergman B, Bullinger M, Cull A, Duez NJ, et al. The European Organization for Research and Treatment of cancer QLQ-30: a quality-of-life instrument for use in international clinical trials in oncology. J Natl Cancer Inst. 1993; 85:365-76.

24. Arraras JI, Kuljanic-Vlasic K, Bjordal K, Yun YH, Efficace F, Holzner B, et al. EORTC QLQ-INFO26: a questionnaire to assess information given to cancer patients a preliminary analysis in eight countries. Psychooncology. 2007;16:249-54

25. K. Bjordal, E. Hammerlid, M. Ahlner-Elmqvist, et al. Quality of life in head and neck cancer patients: validation of the EORTC QLQ-H\&N35. J. Clin. Oncol. 1999; 17:1008-1019

26. Sanders GD, Neumann PJ, Basu A, et al. Recommendations for Conduct, Methodological Practices, and Reporting of Cost-effectiveness Analyses: Second Panel on Cost-Effectiveness in Health and Medicine. JAMA. 2016; 316:1093-1103.

27. Shiroiwa T, Fukuda T, Ikeda S, Shimozuma K. QALY and productivity loss: empirical evidence for "double counting." Value Health. 2013; 16:581-587

28. Sarah J. Whitehead, Shehzad Ali, Health outcomes in economic evaluation: the QALY and utilities, British Medical Bulletin. 2010; 96:5-21.

29. Devleesschauwer, B., Havelaar, A.H., Maertens de Noordhout, C. et al. Int J Public Health. 2014; 59: 565.

30. Franco Sassi, Calculating QALYs, comparing QALY and DALY calculations, Health Policy and Planning. 2006; 21: 402-408.

31. Voltzke, K.J., Lee, YC.A. Zhang, ZF. et al. Cancer Causes Control.2018; 29: 619.

32. Hashibe M. Risk factors: tobacco and alcohol. In: Olshan AF (ed) Epidemiology, pathogenesis, and prevention of head and neck cancer. Springer. 2010; 65-85

33. Stingone JA, Funkhouser WK, Weissler MC, Bell ME, Olshan AF. Racial differences in the relationship between tobacco, alcohol, and squamous cell carcinoma of the head and neck. Cancer Causes Control. 2013; 24:649-664.

34. R. K. C. Ngan, H. H. Y. Yiu, W. H. Lau, S. Yau, F. Y. Cheung, T. M. Chan, C. H. Kwok, C. Y. Chiu, S. K. Au, W. Foo, C. K. Law, K. C. Tse, Combination gemcitabine and cisplatin chemotherapy for metastatic or recurrent nasopharyngeal carcinoma: report of a phase II study, Annals of Oncology. 2002; 13:12521258.

35. W. Budach, E. Bolke, K. Kammers, P.A. Gerber, K. Orth, S. Gripp, et al. Induction chemotherapy followed by concurrent radio-chemotherapy versus concurrent radio-chemotherapy alone as treatment of locally advanced squamous cell carcinoma of the head and neck (HNSCC): a metaanalysis of randomized trials. Radiother Oncol. 2016; 118:238-243

36. A.M. Chen, M.E. Daly, D.G. Farwell, E. Vazquez, J. Courquin, D.H. Lau, et al. Quality of life among longterm survivors of head and neck cancer treated by intensity-modulated radiotherapy. JAMA Otolaryngol Head Neck Surg. 2014;140:129-133

37. B. Zhang, Z. Mo, W. Du, et al. Intensity-modulated radiation therapy versus 2D-RT or 3D-CRT for the treatment of nasopharyngeal carcinoma: a systematic review and meta-analysis. Oral Oncol. 2015; 
51: 1041-1046

38. K. Saarilahti, M. Kouri, J. Collan, et al. Intensity modulated radiotherapy for head and neck cancer: evidence for preserved salivary gland function. Radiother Oncol. 2005;74:251-258

39. E.H.N. Pow, D.L.W. Kwong, A.S. McMillan, M.C.M. Wong, J.S.T. Sham, L.H.T. Leung, et al. Xerostomia and quality of life after intensity-modulated radiotherapy vs. conventional radiotherapy for earlystage nasopharyngeal carcinoma: initial report on a randomized controlled clinical trial. Int J Radiat Oncol Biol Phys. 2006; 66:981-991.

40. A.W. Lee, W.T. Ng, L.L. Chan, W.M. Hung, C.C. Chan, H.C. Sze, O.S. Chan, A.T. Chang, R.M. Yeung. Evolution of treatment for nasopharyngeal cancer-success and setback in the intensity-modulated radiotherapy era. Radiother. Oncol. 2014;110:377-384

41. Adler NE, Boyce WT, Chesney MA, Folkman S, Syme SL. Socioeconomic Inequalities in Health: No Easy Solution. JAMA. 1993; $269: 3140-3145$.

42. Stanford-Moore, G., Bradshaw, P.T., Weissler, M.C. et al. Cancer Causes Control. 2018; 29: 863.

43. Gyllensten, H., Koinberg, l., Carlström, E. et al. Support Care Cancer. 2019; 27: 1825.

44. FM Fang, $C$ Herng-Chia, $\mathrm{K}$ Wen-Rei, et al. Health-related quality of life for nasopharyngeal carcinoma patients with cancer-free survival after treatment. Int J Radiat Oncol Biol Phys. 2002; 53:959-968.

\section{Figures}




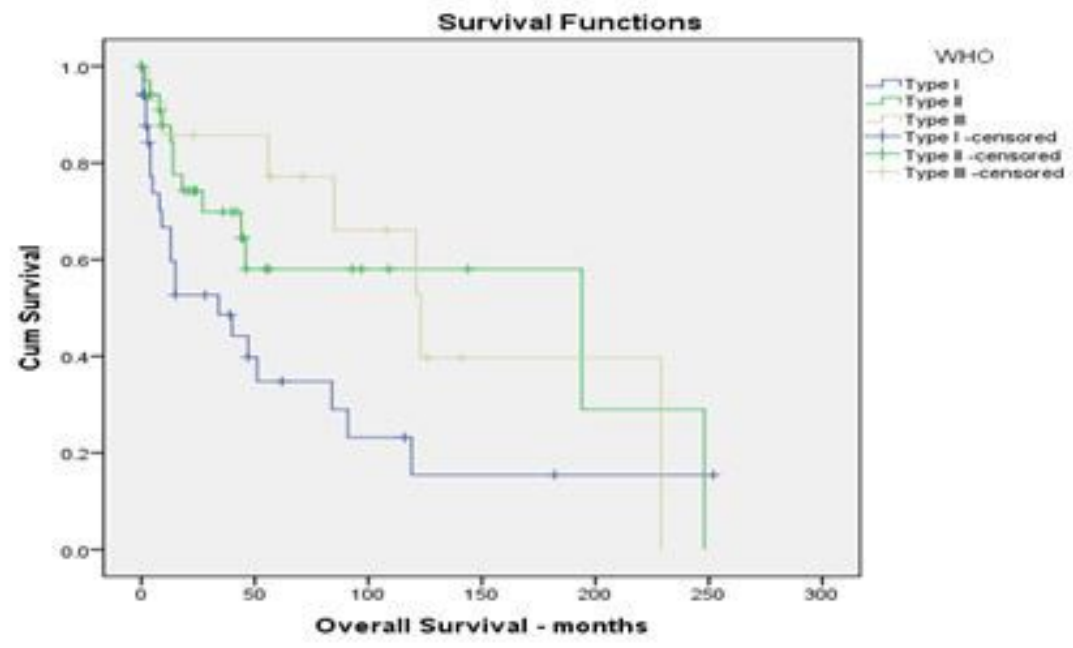

Number of NCP at Risk categorized by WHO Types

\begin{tabular}{|l|r|r|r|r|r|r|r|}
\hline Type I & 34 & 7 & 4 & 2 & 1 & 0 & 0 \\
\hline Type II & 35 & 8 & 4 & 3 & 2 & 1 & 0 \\
\hline Type III & 13 & 10 & 6 & 1 & 0 & 0 & 0 \\
\hline
\end{tabular}

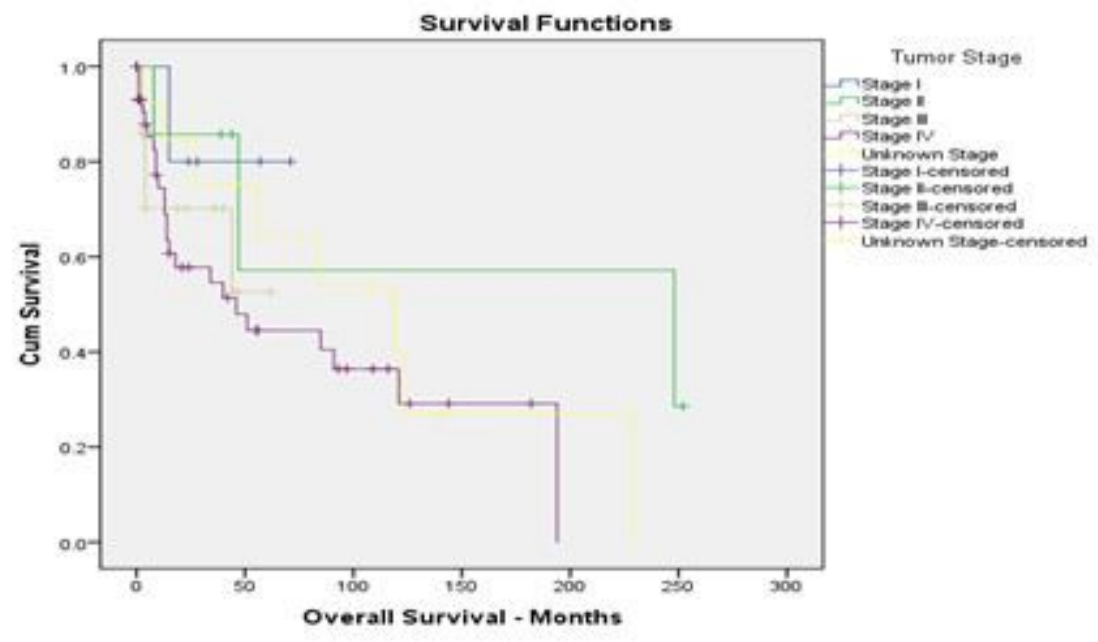

Number of NCP at Risk categorized by Tumor Stage Types

\begin{tabular}{|l|r|r|r|r|r|r|r|}
\hline Stage I & 4 & 2 & 0 & 0 & 0 & 0 & 0 \\
\hline Stage II & 7 & 2 & 1 & 1 & 1 & 0 & 0 \\
\hline Stage III & 13 & 1 & 0 & 0 & 0 & 0 & 0 \\
\hline Stage IV & 44 & 13 & 7 & 2 & 0 & 0 & 0 \\
\hline $\begin{array}{l}\text { Stage } \\
\text { Unknown }\end{array}$ & 12 & 7 & 4 & 1 & 0 & 0 & 0 \\
\hline
\end{tabular}

\section{Figure 1}

The Kaplan- Meier overall survival curves for the study population by tumor histology (WHO types) and by tumor stages. NCP, nasopharyngeal carcinoma; WHO, World Health Organization. 


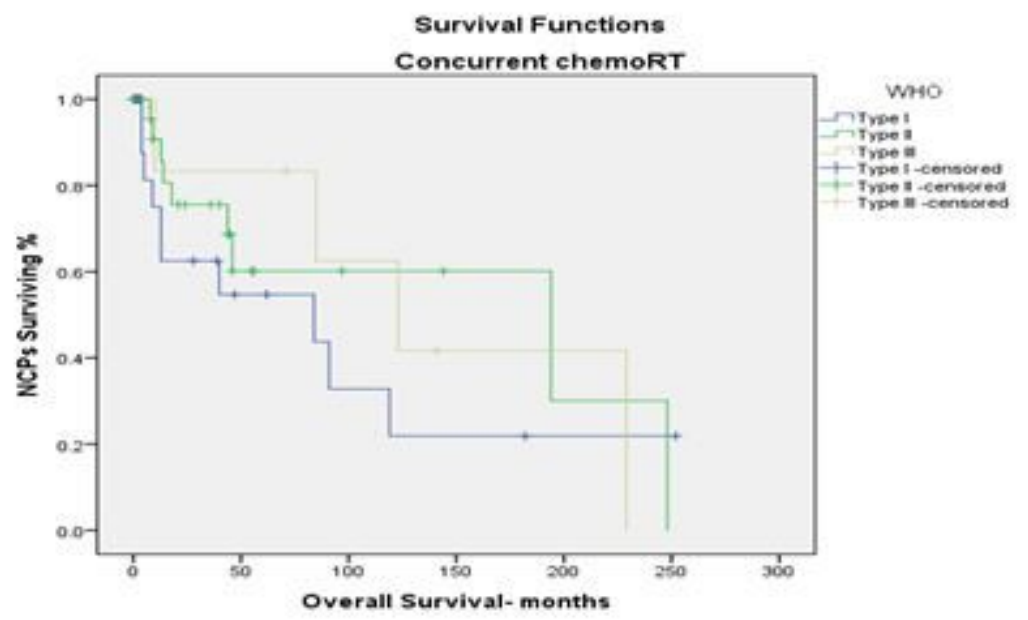

Number of NCP treated concurrent chemoRT at Risk categorized by WHO Types

\begin{tabular}{|l|l|l|l|l|l|l|l|}
\hline Type I & 8 & 2 & 0 & 0 & 0 & 0 & 0 \\
\hline Type II & 7 & 2 & 0 & 0 & 0 & 0 & 0 \\
\hline Type III & 2 & 1 & 0 & 0 & 0 & 0 & 0 \\
\hline
\end{tabular}

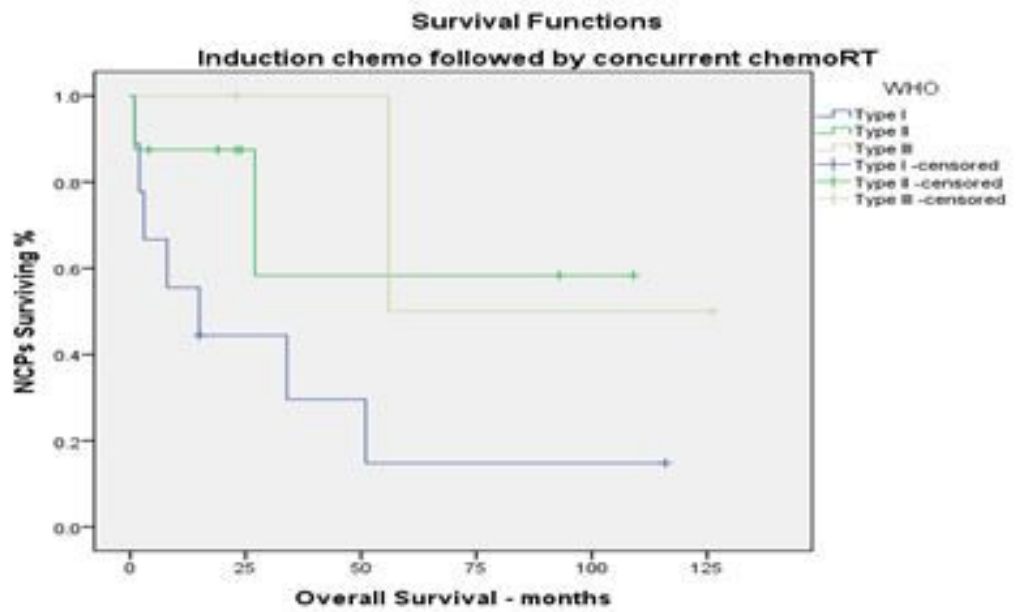

Number of NCP treated by induction chemo followed by concurrent chemoRT at Risk categorized by WHO Types

\begin{tabular}{|l|r|r|r|r|r|r|}
\hline Type I & 20 & 10 & 6 & 5 & 3 & 2 \\
\hline Type II & 23 & 13 & 6 & 4 & 3 & 2 \\
\hline Type III & 5 & 4 & 4 & 3 & 3 & 2 \\
\hline
\end{tabular}

\section{Figure 2}

The Kaplan- Meier overall survival curves for NCPs treated with concurrent chemoRT vs. NCPs treated with induction chemo followed by concurrent chemoRT. NCP, nasopharyngeal carcinoma; WHO, World Health Organization; chemoRT, chemoradiation; chemo, chemotherapy. 